\title{
OBSIDIANAS EN LA PAYUNIA (SUR DE MENDOZA, ARGENTINA): PATRONES DE DISTRIBUCIÓN E IMPLICANCIAS EN LA OCUPACIÓN REGIONAL
}

\author{
LAURA SALGÁN", ADOLFO GIL" Y GUSTAVO NEME**
}

\begin{abstract}
RESUMEN
En este trabajo se presentan los resultados obtenidos del análisis tecnológico de artefactos de obsidiana procedentes del campo volcánico de La Payunia (sur de Mendoza, Argentina). Estudios previos en el centro oeste argentino y Chile central abordaron desde una escala macro regional, el rol las fuentes de obsidiana dentro de las redes de interacción y movilidad humana en diferentes escalas temporales. En La Payunia se identificaron dos fuentes de obsidiana, ubicadas en áreas con características ambientales e historia ocupacional diferentes: fuente El Peceño en área el Nevado y fuente Payún Matrú en área El Payén. Se explora desde escalas meso y micro espaciales, y sobre la base de estudios tecnológicos de artefactos caracterizados por medio de fluorescencia de rayos-X (XRF), las estrategias de abastecimiento y posibles rangos de acción humana en el extremo sur de Mendoza, durante la segunda mitad del Holoceno tardío. Los resultados sugieren marcadas diferencias en el modo uso estas fuentes, así como en la amplitud y direccionalidad de los territorios explotados.
\end{abstract}

PALABRAS CLAVE: obsidiana, nordpatagonia, estrategias de aprovisionamiento, Holoceno tardío, cazadores-recolectores.

\section{OBSIDIAN IN LA PAYUNIA (SOUTH MENDOZA, ARGENTINA): SPATIAL PATTERN AND ITS IMPLICATIONS IN THE REGIONAL ARCHAEOLOGICAL RECORD}

\footnotetext{
ABSTRACT

This paper presents the results of technological analysis of obsidian artifacts from the Volcanic Field of La Payunia (south of Mendoza, Argentina). Previous studies in west central Argentina and central Chile discussed the role of obsidian sources in a regional large scale, interaction networks and human mobility in different scales. In La Payunia two obsidian sources have been identified and located in areas with different environmental characteristics and occupational history. Source El Peceño in the Nevado

Museo de Historia Natural de San Rafael (ANPCYT). Parque Mariano Moreno s/n 5600, San Rafael, Mendoza. Argentina mlaurasalgan@gmail.com

* CONICET - Museo de Historia Natural de San Rafael. Parque Mariano Moreno s/n 5600, San Rafael, Mendoza. Argentina adolfogil@arqueologíamendoza.com, gustavoneme@arqueologiamendoza.com
} 
area and source Payún Matrú in El Payén area. We explore sourcing strategies and potential home-range in the south of Mendoza from middle and small spatial scales, and on the basis of technological studies of artifacts characterized by X-ray fluorescence (XRF), in the second half of the Late Holocene. The results suggest marked differences in the use of obsidian sources, as well as the extent and directionality of the exploited areas.

KEY WORDS: obsidian, North Patagonia, procurement strategies, late Holocene, hunter gatherer.

\section{INTRODUCCIÓN}

La intensidad de uso y la distancia a las fuentes de materias primas líticas es información válida para entender aspectos de la organización tecnológica y direccionalidad de los vínculos entre las poblaciones humanas (Renfrew 1977; Charlin 2009). Por su estructura geoquímica la obsidiana es un indicador sensible que permite abordar estos temas (Glascock et al. 1998; Shackley 2005). Recientemente Giesso et al. (2011) proponen un patrón macro regional que abarca el centro occidente argentino y Chile central, en el cual se destaca la utilidad de la obsidiana para diferenciar la frecuencia con la que fue utilizada cada fuente en los diferentes sectores de la región. Asimismo, se abordaron aspectos temporales en el uso de esta materia prima y la incorporación de las canteras dentro de los circuitos de producción y circulación a lo largo del tiempo (Durán et al. 2004; Neme y Gil 2008). Estos trabajos, apuntaron a resolver problemas de escala macro espacial, sin considerar el papel que pudieron jugar los aspectos tecnológicos en los patrones encontrados. En el presente trabajo exploramos una escala espacial menor, la región de La Payunia, haciendo énfasis en aspectos tecnológicos sobre el uso de la mencionada materia prima.

Gil (2000, 2006), en base a características ambientales divide a la región de La Payunia en dos sub áreas denominadas Área El Nevado (sector norte, $A E N$ ) y Área El Payén (sector sur, AEP), proponiendo un modelo de uso diferencial de ambos. La Payunia es una región volcánica extra cordillerana que presenta un marcado contraste ambiental tanto con la planicie oriental como con el piedemonte y la cordillera (Gil 2000, 2002, 2006; Neme 2002a, 2002b, 2007; Neme y Gil 2008). El registro arqueológico muestra algunas diferencias entre ambas, principalmente en cuanto a estrategias de organización tecnológica, movilidad e historia ocupacional. La región habría sido ocupada en forma efectiva, en el sentido de Borrero (1994-1995) hacia la segunda mitad del Holoceno tardío, ca. 2.000 años AP y hasta tiempos históricos (Gil 2002, 2006). En ambas áreas la obsidiana ocupa el segundo lugar en orden de frecuencia respecto del total de materias primas utilizadas, estudios previos permiten estimar un 4 $\%$ del total en AEN (basaltos 1\%; obsidianas 4\%; y silíceas 95\%), mientras que en AEP representa un 10\% del total (basaltos 3\%; obsidianas 10\%; silíceas 87\%) (Gil 2006). AEN tendría ocupaciones más estables y redundante que las de AEP, estas diferencias en la movilidad y uso del espacio podrían implicar variaciones en la organización tecnológica al menos durante los últimos 2.000 años AP.

En este trabajo, se propone explorar si dicha diferenciación se refleja en la forma que fue utilizada la obsidiana. Para ello se emplean los resultados de análisis de fluorescencia de rayos $\mathrm{X}$ (XRF) y activación neutrónica (INAA), realizados sobre 163 artefactos prevenientes de 11 sitios arqueológicos de La Payunia (Fig. 1). Con dicha información, generada en el marco de un proyecto mayor (Durán et al. 2004; Giesso et al. 2011; Barberena et al. 2011), se discute la direccionalidad de las tendencias, aspectos de los rangos de acción y las formas de aprovisionamiento de esta materia prima entre los diferentes ambientes explotados.

\section{Las obsidianas en nordpatagonia}

La variabilidad en el uso de obsidianas en el sur de Mendoza (Nordpatagonia) ha sido un tema fuertemente abordado en los últimos cinco años (Durán et al. 2004; De Francesco et al. 2006; Giesso et al. 2011; Barberena et al. 2011). Las discusiones se enfocaron principalmente en la detección y caracterización de las fuentes de obsidiana, así como en los rangos de explotación e intensidad de uso de las mismas. Estos trabajos permitieron diferenciar siete fuentes de obsidiana (Tabla 3; Fig. 
1) entre el sur de Mendoza y norte de Neuquén. Las caracterizaciones geoquímicas de estas fuentes están desarrolladas detalladamente en Giesso et al. (2011) y Barberena et al. (2011).

En La Payunia han sido detectadas dos fuentes de obsidianas, El Peceño (AEN) y Payún Matrú (AEP) (Fig. 1), ésta última solo conocida mediante la caracterización de muestras obtenidas en el área por intervención de terceros. El resto de las fuentes se localizan en cordillera con las excepciones de
Huenul y probablemente La Bandera, las cuales fueron caracterizadas como fuentes secundarias (Durán et al. 2004; Giesso et al. 2011; Barberena et al. 2011; Fig. 1). Los trabajos previos mostraron también diferencias en cuanto a la disponibilidad, calidad y accesibilidad de las obsidianas entre las distintas canteras (Giesso et al. 2011). Estas diferencias parecen haber funcionado como limitantes en el uso y dispersión de las obsidianas provenientes del sur de Mendoza. De este modo, las fuentes locali-

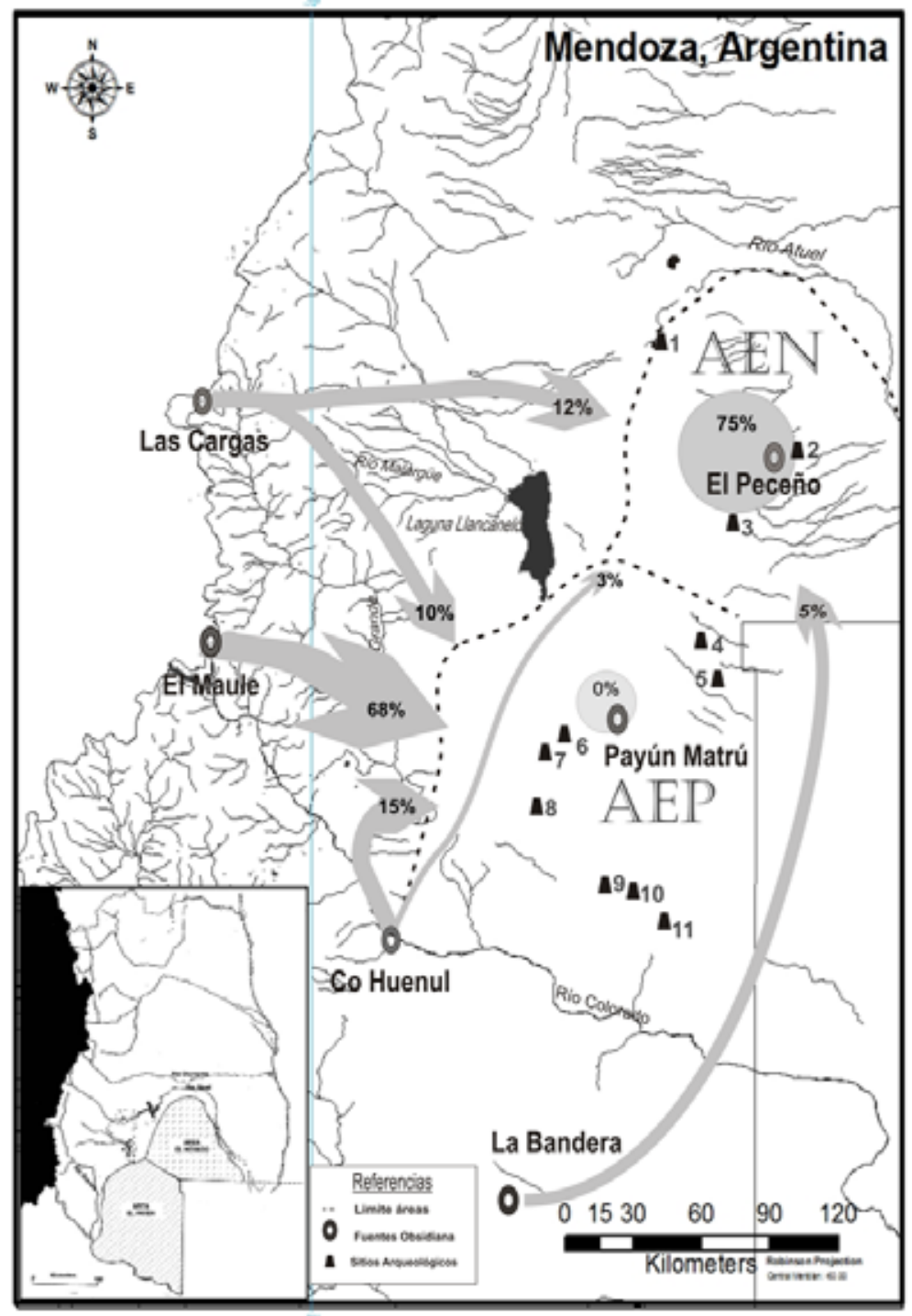

Fig. 1. Localización de las fuentes y sitios mencionados en este trabajo. Notas: AEN: Área El Nevado; AEP: Área El Payén; 1- Arbolito; 2- Puesto Ortubia; 3- Cupertino; 4- Cueva Delerma; 5- La Peligrosa; 6- ALPA Este; 7- Loma Alta; 8- Agua de Pérez; 9- Rincón Blanco; 10- PRC Tren; 11- PRC. 
zadas en cordillera (El Maule, Las Cargas) tendrían una disponibilidad temporal reducida (no se puede acceder en invierno), menor accesibilidad (altura y topografía), pero mayor cantidad y disponibilidad de materia prima (Giesso et al. 2011).

\section{CONSIDERACIONES TEÓRICO METODOLÓGICAS}

En este estudio las estrategias de aprovisionamiento y utilización de materias primas líticas son analizadas en relación con sus costos de obtención y los beneficios que proporcionan (Franco 2004). En áreas con recursos líticos abundantes, es posible que algunos de ellos sean preferidos para determinadas tareas o que existan variaciones en la calidad de los mismos a través del espacio (Callahan 1979; Nami 1992; Aragón y Franco 1997), lo que también puede influir en las estrategias seguidas por los cazadores-recolectores (Amick 1994). La relación entre la distancia a la fuente de aprovisionamiento lítico y la forma de acceso a dicha fuente (directo o indirecto) no es simple ni sigue un patrón lineal (Franco 2004). Es posible que determinadas rocas sean preferidas por otros valores distintos a los tecnológicos, tales como estéticos o religiosos (en el sentido de Gould 1980; Flegenheimer y Bayón 1999), lo cual puede ser demostrado si se usa un marco basado en la relación costo/beneficio.

La distribución espacial de las materias primas líticas, es un indicador utilizado para estudiar el rango de acción de las poblaciones y el contacto o interacción entre ellas (Renfrew 1977; Meltzer 1989; Beck et al. 2002). En este sentido, las rocas de disponibilidad local son comúnmente consideradas de acceso directo $e$ indicadoras de rangos de acción (Binford 1979), en cambio las fuentes no-locales o exóticas son interpretadas o entendidas como resultados de interacción entre poblaciones, y por consiguiente, del intercambio de recursos entre ellas (Meltzer 1989). El concepto de aprovisionamiento directo fue acuñado por Binford (1979). Sobre la base de información etnoarqueológica, Binford diferencia dos tipos de estrategias de aprovisionamiento lítico de acuerdo con los costos implicados: "incluido" ("embedded") versus "aprovisionamiento directo" ("direct procurement strategies") (Binford 1979: 259). El primero de los casos es entendido como aprovisionamiento incidental y hace referencia a la obtención de materias primas en el transcurso del desarrollo de otras tareas, principalmente de subsistencia. Por "aprovisionamiento directo" se refiere a los movimientos de una población en el ambiente con el propósito exclusivo de obtener materias primas para la confección de instrumentos. Para Binford (1979), raramente se hacen viajes con el único propósito de obtener materias primas. Por este motivo, sostiene que las proporciones de materias primas recuperadas en un sitio son básicamente una función de la escala del hábitat que está siendo explotado y no implica costo alguno, o los mismos serían muy bajos. Numerosos autores discuten este último punto (Gould y Saggers 1985; Bamforth 1991; Franco 2004). Ingbar (1994) demostró el efecto de la movilidad y la tasa de reemplazo de instrumentos sobre el porcentaje de materias primas del registro arqueológico, llegando a la conclusión de que las mismas reflejan el rango de acción mínimo del grupo. Kuhn (2004) por su parte sostiene que la relación entre la distancia de transporte y la historia de vida de los artefactos es un indicador más adecuado, en tanto nos informa sobre las transformaciones y usos de los artefactos entre las fuentes y los lugares de descarte.

Los factores a tener en cuenta según Kuhn (2004) son: disponibilidad de recursos en el ambiente (en términos de abundancia, concentración, calidad o valor nutritivo, predictibilidad, entre otros), los circuitos de movilidad y explotación y la relación con otros grupos. Renfrew (1977) desde una perspectiva regional, propuso un modelo cuantitativo de distribución espacial de las materias primas líticas que considera la frecuencia y abundancia de los artefactos según la distancia a la fuente. En caso de un acceso directo a las fuentes, el modelo sostiene un decrecimiento en la frecuencia de los artefactos a medida que aumenta la distancia a los lugares de aprovisionamiento, lo que forma una curva de caída o fall-off (se espera en variables como: abundancia, tamaño y grado de desgaste).

Por su parte la adquisición indirecta de recursos (incluyendo materias primas y artefactos líticos, así como otros tipos de recursos utilitarios y no-utilitarios) involucra varios mecanismos de transferencia de bienes, tales como: el intercambio, la movilidad de individuos o familias, la migración y la conquista, entre otros (Meltzer 1989). Meltzer (1989) señala que en la mayoría de los casos, los estudios arqueológicos han abordado la problemática 
Tabla 1. Expectativas arqueológicas de los conjuntos artefactuales según el modo de aprovisionamiento.

\begin{tabular}{|c|c|c|}
\hline EXPECTATIVAS ARQUEOLÓGICAS & ACCESO DIRECTO & ACCESO INDIRECTO \\
\hline Frecuencia de materia prima en el conjunto & Alta & Baja \\
\hline Representación de estadios de manufactura & Todas las etapas & Predominio de etapas finales \\
\hline Clases artefactuales & Amplio & Restringido \\
\hline Tamaño de los artefactos & Variable & Muy pequeños -pequeños \\
\hline Porcentaje de Corteza & Alto & Bajo \\
\hline Diversidad estilística en diseño de instrumentos & Alta & Baja \\
\hline
\end{tabular}

desde la consideración de los materiales llamados "exóticos". Discute los correlatos materiales de cuatro variables que comúnmente se consideran para diferenciar aprovisionamiento directo del indirecto: la presencia-ausencia de materias primas exóticas, la cantidad y el tipo de artefactos manufacturados en materias primas exóticas, la uniformidad estilística y la secuencia de reducción de los artefactos.

Las expectativas planteadas por los modelos descriptos se resumen en las Tablas 1 y 2 . En el acceso directo, se espera una alta frecuencia de la fuente dentro del conjunto y un elevado índice de corteza. Se espera encontrar representadas las etapas finales de reducción de instrumentos, lo que implica: lascas de reactivación y mantenimiento y/o reutilización de aquellos (Charlin 2009). También siguiendo a Meltzer (1989) se podría esperar cierta diversidad estilística en el diseño de determinados instrumentos, como puntas.

En caso de un acceso indirecto al recurso, se espera que se encuentren representadas las distintas etapas de la secuencia de manufactura (aunque predominen las etapas finales por conservación de la materia prima), presencia de núcleos agotados, lascas y artefactos con corteza (Franco 2007; Charlin 2009). Escaza representatividad en el conjunto y un uso restringido de las materias primas a determinadas clases de instrumentos, además de desechos con rastros de uso.
A partir de estas expectativas se compara la utilización de las distintas fuentes en las áreas analizadas, en cuanto a:

1- frecuencia de las diferentes fuentes de obsidiana 2- clases artefactuales representadas

3- presencia de corteza

4- grado de reducción ponderado según la densidad de negativos de lascados (o extracciones previas) en la cara dorsal de los desechos de talla (Ingbar et al. 1989).

\section{MATERIALES Y MÉTODOS}

Se procesaron 163 especímenes, sus frecuencias por sitio y resultados se detallan en el Tabla 3 . Las muestras fueron procesadas en el MURR siguiendo la metodología para INAA descripta en Durán et al. (2004) y para XRF en Giesso et al. (2011). La información geoquímica de los artefactos procesados corresponde a la publicados en Giesso et al. (2011), para la región bajo estudio. El muestreo de artefactos intentó buscar variabilidad macroscópica regional en las obsidianas del centro occidente argentino, por lo cual en esta etapa no se implementó un criterio de representatividad por sitios. Las muestras corresponden a cuatro sitios arqueológicos en depósitos estratigráficos y siete áreas superficiales donde se realizaron muestreos a través de recolecciones sistemáticas (transectas

Tabla 2. Expectativas arqueológicas en artefactos líticos.

\begin{tabular}{|c|l|l|}
\hline CLASES ARTEFACTUALES & \multicolumn{1}{|c|}{ ACCESO DIRECTO } & \multicolumn{1}{|c|}{ ACCESO INDIRECTO } \\
\hline NÚULEOS & Agotados, formas estandarizadas. & Ausentes o muy explotados. \\
\hline DESECHOS DE TALLA & $\begin{array}{l}\text { Alta variabilidad y frecuencia, } \\
\text { presentes todas las etapas aunque } \\
\text { prevalecen las finales. }\end{array}$ & $\begin{array}{l}\text { Baja variabilidad, prevalecen etapas } \\
\text { finales y de reactivación de artefactos. }\end{array}$ \\
\hline INSTRUMENTOS & Alta variabilidad de tipos artefactuales. & $\begin{array}{l}\text { Alta frecuencia en relación a otras } \\
\text { clases artefactuales. Baja variabilidad } \\
\text { en tipos. Artefactos con corteza. }\end{array}$ \\
\hline
\end{tabular}




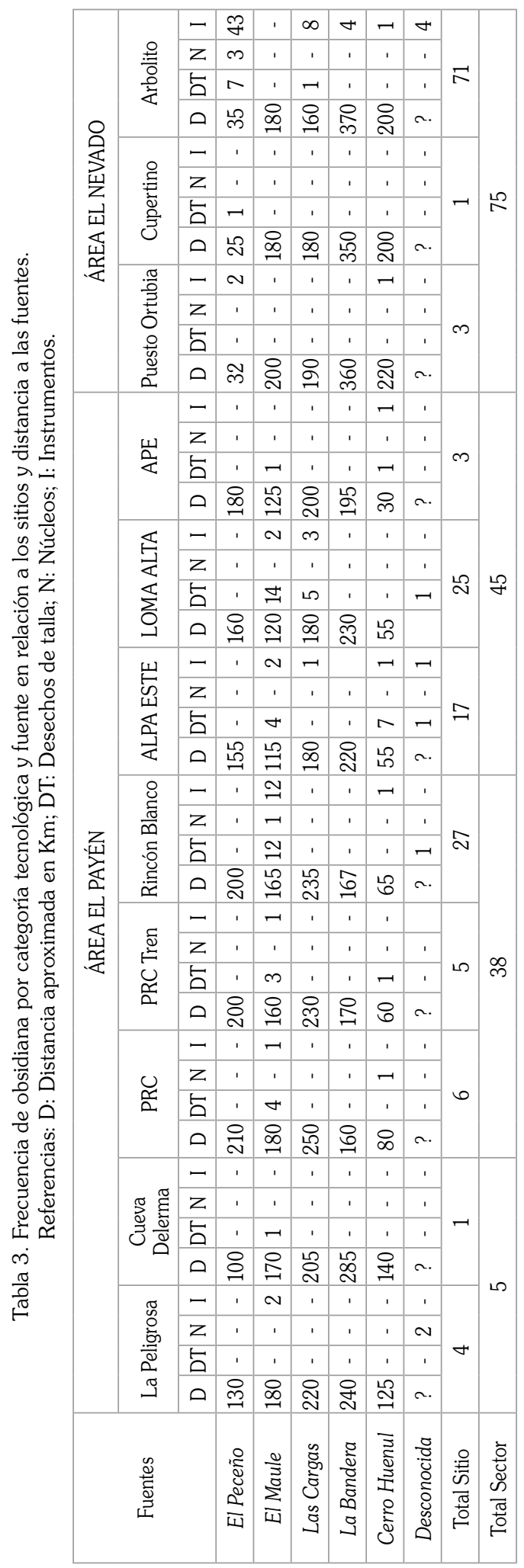

y recolecciones controladas) (Tabla 3). Los sitios estratificados fueron excavados por Gil (2006); dos se localizan en AEN (Puesto Ortubia y Cupertino) y dos en AEP (La Peligrosa y Cueva Delerma). Poseen una cronología correspondiente a la última mitad del Holoceno tardío, con excepción de Cueva Delerma, cuyos fechados basales son de ca. 7000 años AP y corresponden a una ocupación efímera del sitio. Por su parte el registro superficial fue generado bajo una perspectiva distribucional y mayormente detallado en Gil y Neme (2006). El 64\% de los artefactos analizados $(n=154)$ proviene de contextos superficiales, lo que es similar a un escenario arqueológico de patrones del Holoceno tardío (Borrero 2002).

Los análisis tecno-morfológicos de los especímenes se profundizaron a posteriori del análisis geoquímico, siguiendo definiciones y criterios principalmente propuestos por Aschero (1975, 1983). En desechos de talla se calculó su grado de reducción siguiendo el modelo de Ingbar et al. (1989), el mismo es útil como indicador de reducción a partir de la densidad de negativos de lascados (o extracciones previas) en la cara dorsal de la lasca (Odell 2003; Charlin 2007).

\section{TENDENCIAS ESPACIALES DE LAS OBSIDIANAS EN LA PAYUNIA}

La Figura 2 muestra como se representan en cada región las fuentes de obsidiana, mientras que la figura 3 muestra esta tendencia en relación a la distancia lineal estimada entre los sitios y las fuentes. Estas figuras presentan diferencias entre las áreas de La Payunia. En AEN, al igual que en AEP, se registran cinco fuentes de obsidiana explotadas, pero mientras que en AEN predominan artefactos de obsidiana provenientes de El Peceño, geográficamente incluida en la misma área, en AEP la mayor frecuencia es de El Maule emplazada en la cordillera (Fig. 1). AEN es la única que registra La Bandera y no tiene evidencia de uso de El Maule. En cuanto a la distancia estimada entre las fuentes y los sitios, además del uso marcado de la fuente local El Peceño, en AEN se incorporan en bajos porcentajes fuentes distantes de zonas no cordillerana (Cerro Huenul: $2 \%$ y La Bandera 4\% con distancias entre 200 y $400 \mathrm{~km}$ ) y cordillerana (Las Cargas: 9\%, con un rango de distancia estimado entre 100 y $200 \mathrm{~km}$ ). En AEP la tendencia es inversa a la observada en 


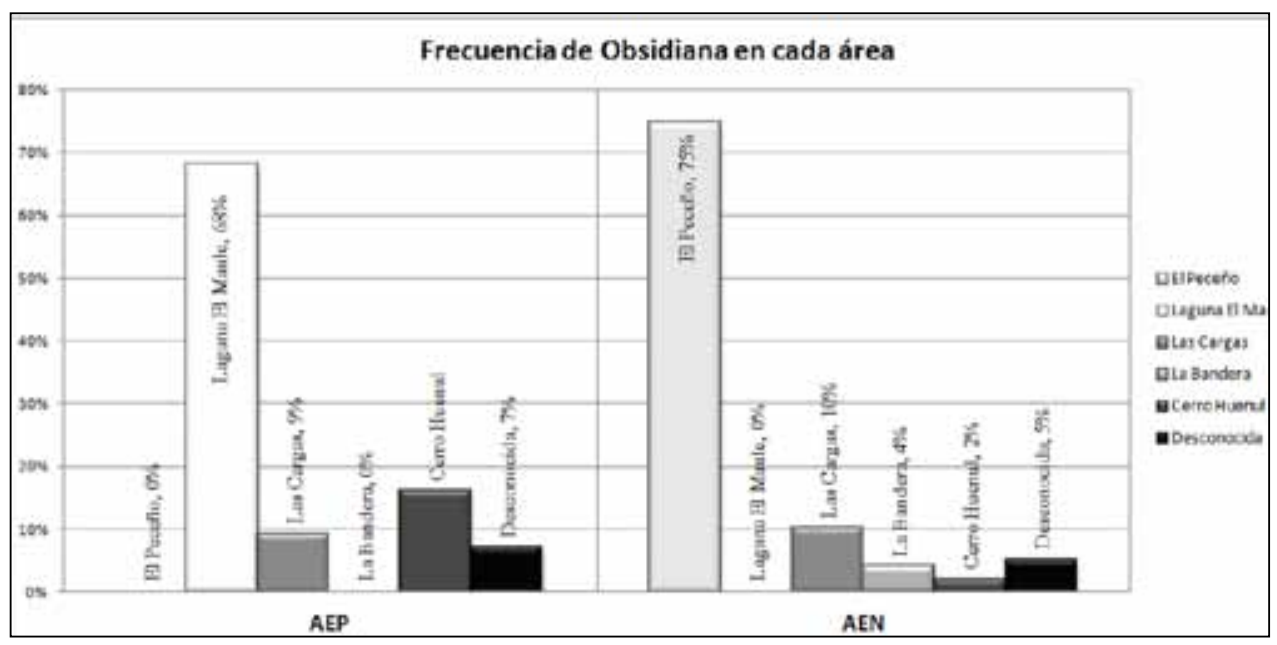

Fig 2. Frecuencia en la representación de cada fuente en las áreas de La Payunia.

AEN pues las fuentes más utilizadas corresponden a cordillera, y superan distancias de $100 \mathrm{~km}$, como es el caso de El Maule (68\%) y Las Cargas (10\%). Se destaca el uso de Cerro Huenul (15\%), ubicada en el sector extra cordillerano, la cual puede ser considerada de disponibilidad local (Meltzer 1989, Civalero y Franco 2003) en relación a los sitios ubicados en el extremo sur de La Payunia (Barberena et al. 2011), con distancias de entre 30 y $100 \mathrm{~km}$ (Fig. 1, Tabla 3).

Las Tablas 4 y 5 señalan el modo en que cada fuente está registrada en las dos áreas. En AEN predomina El Peceño tanto en lascas como en instrumentos y núcleos. Las otras fuentes ingresan como artefactos formatizados, con excepción de Las Cargas que presenta también productos de talla. Esta tendencia es más variable en AEP donde si bien la mayoría de los artefactos se registran sobre obsidiana de El Maule, también hay presencia de núcleos, productos de talla e instrumentos de Huenul, mientras que Las Cargas solo aparece como producto de talla e instrumento.

La proporción de corteza es también significativamente distinta entre ambas áreas (Tabla 6). Tanto en AEN como en AEP, Cerro Huenul presenta una frecuencia significativa de elementos con corteza. En ambas áreas luego sigue la cantera más explotada, mientras que las restantes fuentes de obsidianas en ambos sectores se encuentran sin corteza. Relevamientos superficiales de Cerro Huenul (Durán et al. 2004; Barberena et al. 2011) describen que la materia prima se presenta como clastos medianos y pequeños de depósito secundario, con morfología esférica a sub-redondeada, de excelente

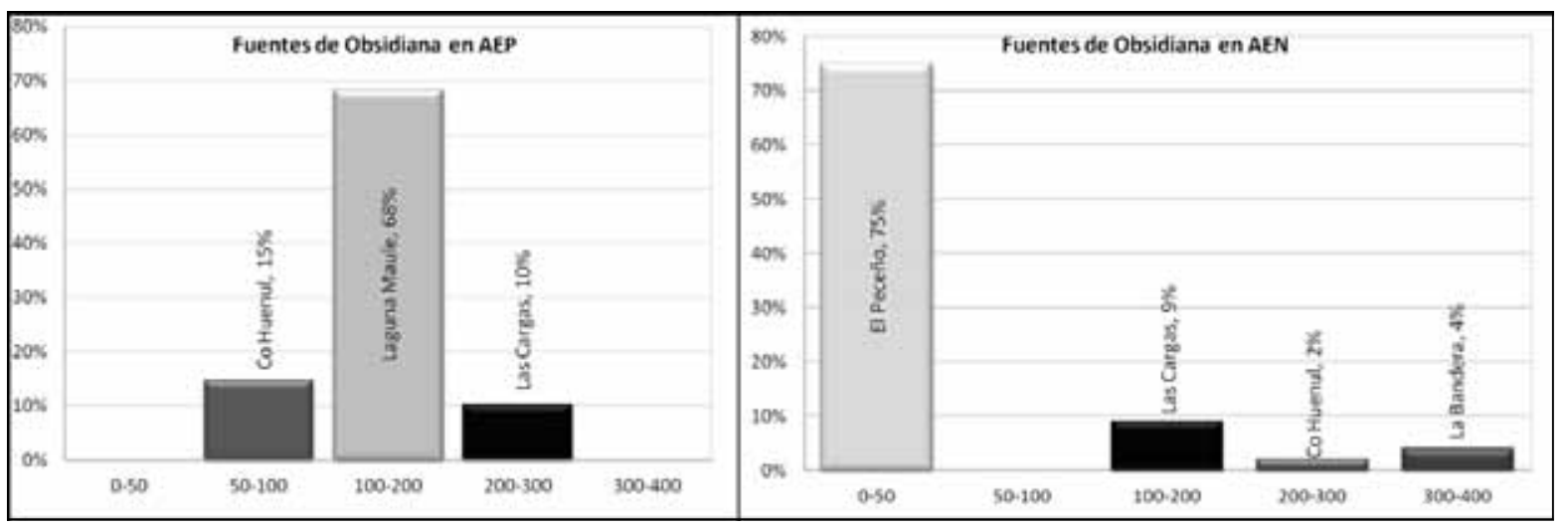

Fig 3. Frecuencia de cada fuente en relación a la distancia estimada. 
Tabla 4. Representación proporcional de cada categoría artefactual por fuente y discriminando las áreas. El valor entre paréntesis corresponde al $n$ total.

\begin{tabular}{|c|c|c|c|c|c|c|c|}
\hline & \multirow{3}{*}{$\begin{array}{c}\text { Cerro Huenul } \\
-\end{array}$} & \multirow{3}{*}{$\begin{array}{c}\text { El Peceño } \\
89 \%(8)\end{array}$} & \multirow{3}{*}{$\begin{array}{c}\text { La Bandera } \\
-\end{array}$} & \multirow{3}{*}{$\begin{array}{c}\text { El Maule } \\
-\end{array}$} & \multirow{3}{*}{$\begin{array}{c}\text { Las Cargas } \\
11 \%(1)\end{array}$} & \multirow{3}{*}{$\begin{array}{c}\text { Desconocida } \\
-\end{array}$} \\
\hline & & & & & & & \\
\hline \multirow{4}{*}{ 学 } & Desecho Talla & & & & & & \\
\hline & Instrumento & $3 \%(2)$ & $71 \%(45)$ & $6 \%(4)$ & - & $13 \%(8)$ & $6 \%(4)$ \\
\hline & Núcleo & - & $100 \%(3)$ & - & - & - & - \\
\hline & $\mathrm{N}$ & 2 & 56 & 4 & - & 9 & 4 \\
\hline \multirow{4}{*}{ 宸 } & Desechos Talla & $16 \%(9)$ & - & - & $67 \%(39)$ & $9 \%(5)$ & $9 \%(5)$ \\
\hline & Instrumento & $11 \%(3)$ & - & - & $71 \%(20)$ & $14 \%(4)$ & $4 \%(1)$ \\
\hline & Núcleo & $50 \%(1)$ & - & - & $50 \%(1)$ & - & - \\
\hline & $\mathrm{N}$ & 13 & - & - & 60 & 9 & 6 \\
\hline
\end{tabular}

Tabla 5. Representación proporcional de cada fuente por categoría artefactual y discriminando las áreas.

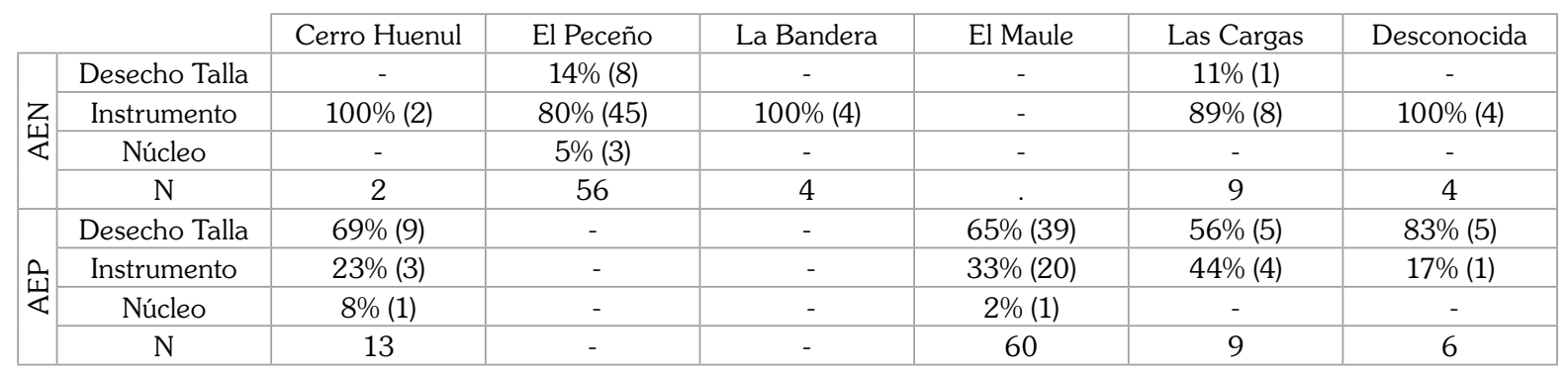

calidad, abundantes y fácilmente transportables. A partir de los estudios de variación morfométrica y el análisis cualitativo de su calidad para la talla, se propuso que el tamaño sería una de las variables consideradas para la selección de los nódulos, limitando el testeo de los mismos en la fuente (Barberena et al. 2011). Si bien esta caracterización es preliminar, se podría esperar que los nódulos sean transportados enteros y que un porcentaje significativo de los artefactos obtenidos presenten remanentes de corteza, lo que es coherente con lo observado en AEP. En el caso de Las Cargas, si bien la fuente presenta una gran extensión espacial (Giesso et al. 2011), los relevamientos superficiales realizados para el presente trabajo dan cuenta de la presencia escasa o nula de corteza en su forma primaria. En su lugar presenta una cubierta en forma de pátina de espesor variable, difícil de identificar como corteza en los conjuntos arqueológicos. Por lo cual se puede pensar que la variabilidad registrada es la esperable, para el modo en que se presenta la obsidiana en la fuente primaria.

Tabla 6. Proporción de corteza en productos de talla de cada área; frecuencia relativa por fuente.

\begin{tabular}{|c|c|c|c|c|c|c|c|}
\hline & & Cerro Huenul & El Peceño & La Bandera & El Maule & Las Cargas & Desconocida \\
\hline \multirow{6}{*}{ 学 } & $0 \%$ & $50 \%$ & $79 \%$ & $100 \%$ & - & $89 \%$ & $75 \%$ \\
\hline & $25 \%$ & $50 \%$ & $9 \%$ & - & - & $11 \%$ & $25 \%$ \\
\hline & $50 \%$ & - & $5 \%$ & - & - & - & - \\
\hline & $75 \%$ & - & $7 \%$ & - & - & - & - \\
\hline & $100 \%$ & - & - & - & - & - & - \\
\hline & $\mathrm{N}$ & 2 & 56 & 4 & . & 9 & 4 \\
\hline \multirow{6}{*}{ 宸 } & $0 \%$ & $23 \%$ & - & - & $72 \%$ & $89 \%$ & $100 \%$ \\
\hline & $25 \%$ & $31 \%$ & - & - & $17 \%$ & $11 \%$ & - \\
\hline & $50 \%$ & $8 \%$ & - & - & $2 \%$ & - & - \\
\hline & $75 \%$ & $15 \%$ & - & - & $8 \%$ & - & - \\
\hline & $100 \%$ & $23 \%$ & - & - & $2 \%$ & - & - \\
\hline & $\mathrm{N}$ & 13 & - & - & 60 & 9 & 6 \\
\hline
\end{tabular}


Tabla 7. Tendencia en el estado de los instrumentos.

\begin{tabular}{|c|c|c|c|c|c|c|c|}
\hline & & Cerro Huenul & El Peceño & La Bandera & El Maule & Las Cargas & Desconocida \\
\hline \multirow{3}{*}{ 学 } & Entero & & $60 \%$ & $50 \%$ & - & $50 \%$ & $25 \%$ \\
\hline & Fracturado & $100 \%$ & $40 \%$ & $50 \%$ & - & $50 \%$ & $75 \%$ \\
\hline & $\mathrm{N}$ & 2 & 45 & 4 & - & 8 & 4 \\
\hline \multirow{3}{*}{ 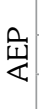 } & Entero & - & - & - & $70 \%$ & - & $100 \%$ \\
\hline & Fracturado & $100 \%$ & - & - & $30 \%$ & $100 \%$ & - \\
\hline & $\mathrm{N}$ & 3 & - & - & 20 & 4 & 1 \\
\hline
\end{tabular}

La Tabla 7 muestra que las fuentes más utilizadas en ambas áreas registran una mayor frecuencia de descarte de instrumentos enteros por sobre los fracturados. En cuanto a los módulos de medida (Tabla 8), en AEN hay una tendencia a módulos espesos y muy espesos, solo encontramos artefactos poco espesos en obsidianas provenientes de la fuente local (El Peceño 5\%). Por el contrario en AEP, si bien los mayores porcentajes corresponden a módulos espesos, los artefactos poco espesos siguen en importancia en las fuentes El Maule (20\%), Las Cargas (33\%) y las desconocidas (17\%). La baja proporción de módulos muy espesos, puede darse como consecuencia del mantenimiento de los artefactos. La Tabla 9 señala la tendencia en relación al módulo tamaño, donde se observa que en ambas áreas predominan los menores tamaños y hay ausencia de piezas medianas y grandes. En AEN predominan los artefactos pequeños y muy pequeños, con excepción de Las Cargas que presenta módulos predominantemente pequeños. En AEP las mayores frecuencias se encuentran en tamaños muy pequeños seguidos por tamaños pequeños. En cuanto a los desechos de talla, para las fuentes más utilizadas en ambas áreas, se encuentran representados los estadios finales de formatización. En el caso de El Maule (AEP), se registra un alto porcentaje de reactivación de filos y lascas de talla bipolar. Esto podría indicar un mantenimiento de filos y un alto aprovechamiento de la materia prima, concordante con una estrategia de economía de materia prima (Franco 2004; Bamforth 1986), idea que deberá ser testeada en futuros trabajos.

Tabla 8. Módulo ancho/espesor de artefactos en cada área; frecuencia relativa a cada fuente.

\begin{tabular}{|c|c|c|c|c|c|c|c|}
\hline & & Cerro Huenul & El Peceño & La Bandera & El Maule & Las Cargas & Desconocida \\
\hline \multirow{5}{*}{ 胥 } & Poco espeso & - & $5 \%$ & - & - & - & - \\
\hline & Espeso & $50 \%$ & $63 \%$ & $75 \%$ & - & $89 \%$ & $75 \%$ \\
\hline & Muy espeso & $50 \%$ & $32 \%$ & $25 \%$ & - & $11 \%$ & $25 \%$ \\
\hline & $\mathrm{N}$ & 2 & 56 & 4 & - & 9 & 4 \\
\hline & & Cerro Huenul & El Peceño & La Bandera & El Maule & Las Cargas & Desconocida \\
\hline \multirow{4}{*}{ 岂 } & Poco espeso & - & - & - & $20 \%$ & $33 \%$ & $17 \%$ \\
\hline & Espeso & $62 \%$ & - & - & $50 \%$ & $56 \%$ & $50 \%$ \\
\hline & Muy espeso & $38 \%$ & - & - & $30 \%$ & $11 \%$ & $33 \%$ \\
\hline & $\mathrm{N}$ & 13 & - & - & 60 & 9 & 6 \\
\hline
\end{tabular}

Tabla 9. Tamaño de artefactos en cada área; frecuencia relativa a cada fuente.

\begin{tabular}{|c|c|c|c|c|c|c|c|}
\hline & & Cerro Huenul & El Peceño & La Bandera & El Maule & Las Cargas & Desconocida \\
\hline \multirow{4}{*}{ 学 } & Muy Pequeño & $50 \%$ & $41 \%$ & $25 \%$ & - & $56 \%$ & $100 \%$ \\
\hline & Pequeño & $50 \%$ & $59 \%$ & $75 \%$ & - & $44 \%$ & - \\
\hline & Mediano & - & - & - & - & - & - \\
\hline & $\mathrm{N}$ & 2 & 56 & 4 & - & 9 & 4 \\
\hline \multirow{4}{*}{ 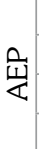 } & Muy Pequeño & $77 \%$ & - & - & $65 \%$ & $100 \%$ & $83 \%$ \\
\hline & Pequeño & $23 \%$ & - & - & $35 \%$ & - & $17 \%$ \\
\hline & Mediano & - & - & - & - & - & - \\
\hline & $\mathrm{N}$ & 13 & - & - & 60 & 9 & 6 \\
\hline
\end{tabular}


Tabla 10. Desechos de talla de cada área; frecuencia relativa de cada fuente. Referencias: Ad. Bifacial: Adelgazamiento bifacial; ${ }^{* 1}$ Reactivación de instrumentos, incluye lascas de reactivación directa e indirecta sensu Aschero (1975, 1983);

*2Las lascas de reactivación de núcleo incluye lascas de flanco de núcleo y tableta de núcleo sensu Aschero (1975, 1983).

\begin{tabular}{|c|c|c|c|c|c|c|c|}
\hline & & Cerro Huenul & El Peceño & La Bandera & El Maule & Las Cargas & Desconocida \\
\hline \multirow{12}{*}{ 案 } & Primaria & - & - & - & - & - & - \\
\hline & Secundaria & - & $13 \%$ & - & - & - & - \\
\hline & Angular & - & $25 \%$ & - & - & $100 \%$ & - \\
\hline & Arista & - & $25 \%$ & - & - & - & - \\
\hline & Plana & - & $13 \%$ & - & - & - & - \\
\hline & Bipolar & - & - & - & - & - & - \\
\hline & Ad. Bifacial & - & - & - & - & - & - \\
\hline & React. Inst & - & - & - & - & - & - \\
\hline & React. Núcleo & - & - & - & - & - & - \\
\hline & No Diferenciada & - & - & - & - & - & - \\
\hline & $\mathrm{N}$ & - & 8 & - & - & 1 & - \\
\hline & & Cerro Huenul & El Peceño & La Bandera & El Maule & Las Cargas & Desconocida \\
\hline \multirow{11}{*}{ 岽 } & Primaria & $22 \%$ & - & - & - & - & - \\
\hline & Secundaria & - & - & - & - & - & - \\
\hline & Angular & $11 \%$ & - & - & $36 \%$ & $40 \%$ & $40 \%$ \\
\hline & Arista & $22 \%$ & - & - & $23 \%$ & $40 \%$ & $20 \%$ \\
\hline & Plana & - & - & - & $5 \%$ & - & - \\
\hline & Bipolar & - & - & - & $3 \%$ & - & - \\
\hline & Ad. Bifacial & $11 \%$ & - & - & & - & - \\
\hline & React. Instr. ${ }^{* 1}$ & - & - & - & $8 \%$ & $20 \%$ & - \\
\hline & React. Núcleo*2 & $22 \%$ & - & - & $5 \%$ & - & - \\
\hline & No Diferenciada & - & - & - & $18 \%$ & - & $40 \%$ \\
\hline & $\mathrm{N}$ & 9 & - & - & 39 & 5 & 5 \\
\hline
\end{tabular}

En la Tabla 11 se presenta la estadística descriptiva de la densidad de extracciones previas en la cara dorsal de los desechos de talla de las distintas fuentes representadas en cada área. Como puede observarse, de acuerdo con los valores de densidad media, AEP presenta un mayor grado de reducción en desechos de talla, sin embargo no hay diferencias significativas entre ambas áreas. Distintos autores plantean que a mayor distancia de la fuente, se espera una mayor intensidad en el uso de la materia prima (Renfrew 1977; Franco 2002). En el caso de AEP cabría esperar, por las distancias de las fuentes representadas (entre 50 y $200 \mathrm{~km}$ lineales), una mayor densidad de extracciones en los artefactos recuperados. Sin embargo, observamos que si bien en ambos con- juntos se da una baja variabilidad en la densidad de extracciones, en AEP encontramos un rango mayor de variación. Esto sugiere que en ambos conjuntos están presentes los primeros estadios de manufactura, desde los estadios iniciales, como lo indican los valores mínimos iguales a cero en ambas áreas (indicando lascas con escasas o nulas extracciones previas, es decir lascas primarias y secundarias en el sentido de Aschero 1983), hasta etapas avanzadas de manufactura con leves diferencias en AEP en los valores máximos. Como se mencionara previamente, los conjuntos analizados fueron muestreados en función de cubrir la mayor variabilidad macroscópica de los sitios, por lo cual será necesario discutir estos resultados a la luz de un muestreo orientado a preguntas tecnológicas.

Tabla 11. Estadística descriptiva de la densidad de extracciones previas en la cara dorsal de los desechos de talla de las distintas fuentes en cada área.

\begin{tabular}{|c|c|c|c|c|c|c|c|}
\hline ÁREA & $\mathrm{N}$ & Media & Mediana & Desvío estándar & Error estándar & Mínimo & Máximo \\
\hline AEN & 9 & 0.27 & 0.17 & 0.24 & 0.08 & 0.00 & 0.65 \\
\hline AEP & 58 & 0.34 & 0.26 & 0.38 & 0.05 & 0.00 & 1.86 \\
\hline
\end{tabular}


Tabla 12. Estadística descriptiva de la densidad de extracciones previas en desechos de talla según procedencia en cada área.

\begin{tabular}{|c|c|c|c|c|c|c|c|c|}
\hline ÁREA & Procedencia & $\mathrm{n}$ & Media & Mediana & $\begin{array}{l}\text { Desvío } \\
\text { estándar }\end{array}$ & Error estándar & Mínimo & Máximo \\
\hline \multirow{2}{*}{ 楚 } & Las Cargas & 1 & 0.28 & - & - & - & - & - \\
\hline & El Peceño & 8 & 0.26 & 0.16 & 0.09 & 0 & 0.65 & 0.65 \\
\hline \multirow{4}{*}{ 岂 } & Desconocidas & 5 & 3.1 & 0.28 & 1.45 & 0.65 & 1.35 & 4.97 \\
\hline & Las Cargas & 5 & 0.62 & 0.33 & 0.73 & 0.33 & 0 & 1.86 \\
\hline & Cerro Huenul & 9 & 0.4 & 0.1 & 0.61 & 0.2 & 0 & 1.71 \\
\hline & El Maule & 39 & 0.26 & 0.25 & 0.2 & 0.03 & 0 & 0.74 \\
\hline
\end{tabular}

En la Tabla 12 se detallan las densidades de extracciones previas en desechos de talla según su procedencia. En AEP se observa mayor variación, siendo la media de las fuentes desconocidas la de mayor densidad de extracciones, seguida por Las Cargas y Cerro Huenul. El Maule presenta valores bajos, y es la clase artefactual con mayores muestras analizadas en el área. En el caso de AEN solo se cuenta con desechos de El Peceño y solo un desecho de Las Cargas, por lo cual deben considerarse como resultados tentativos.

La figura 4 muestra como se presenta la variabilidad en el grado de reducción según la distancia a la fuente en cada área. La mayor variabilidad en densidad de extracciones en AEP se presenta para Cerro Huenul (rango de distancia entre $50-100 \mathrm{~km}$ ) y Las Cargas (con distancias entre 200-300 km). Esto es concordante con la presencia de lascas primarias y de reactivación de núcleos en la primera, y de lascas internas y reactivación de instrumentos en la segunda. En el caso de El Maule (AEP) y El Peceño (AEN), presentan similar densidad de extracción, sin embargo en El Maule se destaca la presencia

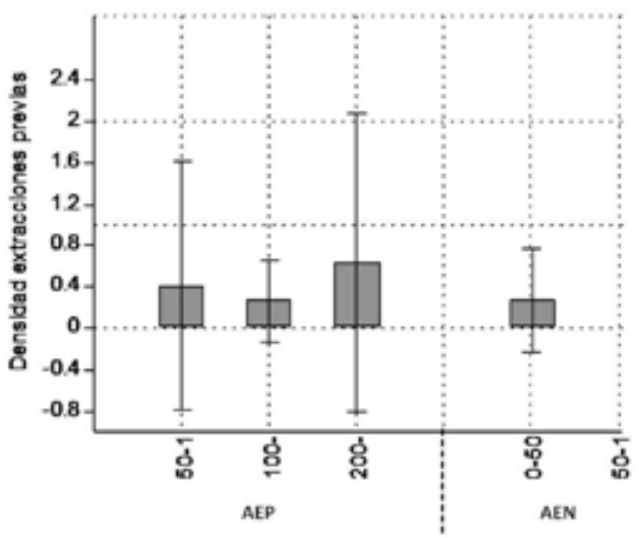

Fig. 4. Frecuencia de densidad de extracciones en AEP y AEN según distancia en $\mathrm{km}$ a la fuente. de lascas de reactivación de núcleos e instrumentos lo que puede responder a diferentes estrategias de conservación de la materia prima.

\section{APROVISIONAMIENTO DE OBSIDIANAS EN LA PAYUNIA}

Los resultados obtenidos a partir de los análisis tecnológicos señalan significativas diferencias entre ambas áreas, las cuales son más notorias en relación al rango de acción. En AEN se observa un mayor uso de fuentes locales, como es la fuente $\mathrm{El}$ Peceño, y entre las menos representadas prevalecen las no cordilleranas. En cambio en AEP, las fuentes más representadas se encuentran a distancias superiores a los $100 \mathrm{~km}$ y provienen de ambientes de cordillera. En cuanto a las fuentes menos representadas, corresponden tanto a cordillera como a ambientes no cordilleranos. Podría considerarse que en ambas áreas el aprovisionamiento de las fuentes mas representadas es directo, con marcadas diferencias en la amplitud de los rangos de acción de las poblaciones.

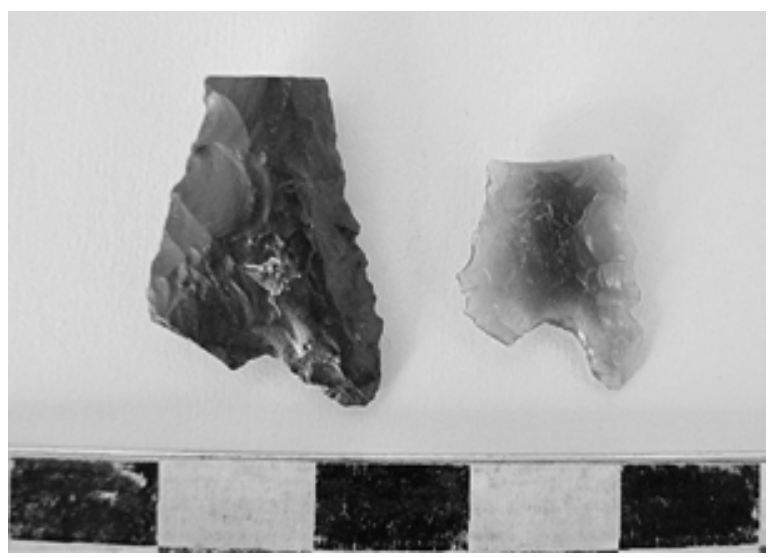

Fig. 5. Instrumentos fragmentados del sitio Arbolito (AEN). 
En este sentido en AEN se observa un marcado uso de la fuente local El Peceño de muy buena calidad para la talla y accesible todo el año (Durán et al. 2004; Giesso et al. 2011). Las clases artefactuales incluyen instrumentos, desechos de talla -en su mayoría correspondientes a las últimas etapas de formatización- y núcleos (5\%). Asimismo, se observa la incorporación de materias primas muy distantes de ambientes preferentemente no cordilleranos, incorporados como instrumentos de tamaños pequeños a muy pequeños y de espesor variable entre espeso y muy espeso, descartados en su mayoría fracturados. Esto podría estar marcando el ingreso de instrumentos terminados de obsidianas distantes o en avanzada etapa de formatización, lo que explicaría la escasa presencia de desechos de talla sobre estas variedades de obsidianas. La única fuente cordillerana representada en AEN es Las Cargas, presente en forma de instrumentos y de lascas angulares correspondientes a tareas finales de formatización. A partir del patrón observado en las fuentes distantes presentes en $\mathrm{AEN}$, es posible sostener la circulación de artefactos formatizados o en avanzada etapa de formatización, lo que se ajusta con lo esperado para modos de aprovisionamiento indirecto.

En AEP, y a diferencia de AEN, encontramos que tanto las fuentes más representadas (El Maule $68 \%$ y Cerro Huenul 15\%), como la de menor representatividad en el conjunto (Las Cargas 10\%), presentan alta variabilidad en las clases artefactuales. En la Tabla 5 se destaca la alta frecuencia de desechos de talla de las distintas fuentes, seguido por instrumentos y núcleos. En desechos de talla (Tabla 10), los tipos con mayor representatividad corresponden a las etapas finales de formatización, seguido por desechos de reactivación de instrumentos de obsidianas El Maule (8\%) y Las Cargas (20\%) y lascas de reactivación de núcleos de Cerro Huenul $(22 \%)$ y El Maule (5\%). Dada la variabilidad de las clases artefactuales y tipos de desechos de talla, se podría sostener la circulación de las distintas variedades de obsidiana en forma de nódulos. Por otro lado, las obsidianas evidencian diferencias en cuanto a los módulos de tamaño y formas de descarte de instrumentos. Cerro Huenul, Las Cargas y Desconocidas presentan una alta frecuencia de módulos espesos y muy espesos, tamaños muy pequeños y descarte de instrumentos fracturados, lo que podría indicar de tareas de mantenimiento de herramientas de larga vida útil. Los instrumentos de El Maule, por su parte, fueron descartados mayoritariamente enteros, con tamaños muy pequeños a pequeños $y$ espesos a muy espesos, si bien hay evidencias de actividades de formatización y mantenimiento de herramientas, ésta sería menos frecuente en la fuente más representada. Estos resultados se condicen con lo esperado para estrategias tendientes a economizar o maximizar el uso de materia prima de alta calidad en el caso de las obsidianas de Las Cargas, Desconocidas y quizás Cerro Huenul (en el sentido de Franco 2004); así como a estrategias vinculadas con situaciones de estrés temporal (Kuhn 1994) donde los instrumentos relacionados con la adquisición de recursos de subsistencia se descartan rápidamente, como es el caso de obsidianas El Maule (Binford 1979; Bamforth 1986; Franco 2004).

Entonces a partir del estudio de los conjuntos artefactuales y los modos de aprovisionamiento de los mismos, se plantea que las estrategias seguidas en ambas áreas parecen responder a distintos modos de uso del espacio y circulación entre AEN y AEP. En AEN se pueden plantear dos modos de acceso a las materias primas: un acceso directo a las fuentes locales y la incorporación de fuentes distantes de modo indirecto, cuya circulación apunta a sectores no cordilleranos de Nordpatagonia, en el caso de Cerro Huenul y La Bandera, y cordilleranos, como es el caso de la fuente Las Cargas. En AEP por su parte, y según las expectativas generadas para cada modo de aprovisionamiento, se puede sostener que las obsidianas más representadas fueron obtenidas de modo directo, lo que implicó el traslado de materias primas de fuentes distantes, provenientes en su mayoría de cordillera (El Maule y Las Cargas). Lo que sugieren una mayor amplitud de los rangos de acción, una prevalencia de circulación Oeste-Este y la necesidad de planificar tanto el uso de materias primas como los respectivos viajes de aprovisionamiento y/o intercambio.

Un punto importante para destacar es que la fuente Payún Matrú, la más próxima para AEP, no cuenta con registro de explotación en la región. En relación a esto Giesso et al. (2011), encuentran que los artefactos con esta señal poseen una distribución con dirección noroeste, estando presentes en dos sitios de cordillera próximos al río Grande (sitios Cañada de Cachi y Cueva de Luna), distantes entre 
75 y $50 \mathrm{~km}$ en línea recta de la fuente, además de un sitio no cordillerano próximo a la Laguna Llancanelo (sitio Llan 29) distante aproximadamente $100 \mathrm{~km}$, todos correspondientes al Holoceno tardío. Por otro lado se menciona la presencia de un artefacto procedente de Payún Matrú, en contextos de ca. 7.200 años AP, en el sitio Gruta El Manzano (Giesso et al. 2011). Esto podría reforzar la hipótesis de que La Payunia formó parte de circuitos de desplazamientos estacionales que alternaban ambientes de altura y planicie (según la idea de Gambier 1985 y promovida por Durán 2000), y/o con la variante de esta misma idea que sostiene que la región pudo ser ocupada de modo intermitente desde las regiones vecinas (Borrero 2002), al menos para mediados de Holoceno y primera mitad del Holoceno tardío.

\section{CONCLUSIÓN}

El modo en que las poblaciones se abastecen de recursos puede ser correlacionado con el rango de acción mínimo de los territorios explotados (Ingbar 1994). Distintos investigadores han remarcado la dificultad de definir modos específicos de aprovisionamiento (Goul y Saggers 1985; Ericson 1984; Meltzer 1989). En base a las expectativas planteadas y al análisis de los artefactos de obsidiana de La Payunia, es posible identificar en la región distintos patrones de explotación de las fuentes de obsidiana durante el Holoceno tardío, así como discutir y establecer conexiones entre diferentes espacios.

En el caso de AEN algunos indicadores sugieren variaciones en los modos de obtención de estas rocas a través del espacio. Así, para la obsidiana de El Peceño se propone un abastecimiento directo, con la incorporación de artefactos formatizados de regiones distantes. En esta área se destaca la presencia de fuentes no cordilleranas, sin embargo trabajos recientes (Giesso et al. 2011) señalan su baja representación en los sitios en una escala espacial amplia. No obstante en AEN, la utilización de dichas fuentes cobra relevancia. Para AEP, dada la variabilidad presente en cuanto a abundancia y clases artefactuales, se sugiere un patrón diferente que incorpora fuentes preferentemente de cordillera, con distancias superiores a los $100 \mathrm{~km}$, y de modo subsidiario obsidianas provenientes de fuentes cercanas como las de Cerro Huenul.
Este primer acercamiento ha permitido evaluar funcionalmente la distribución de obsidianas en la región de La Payunia, permitiendo reconocer usos diferenciales de esta materia prima dentro de dos tipos de ambientes de una misma región. En adelante será necesario realizar análisis de elementos traza con materiales seleccionados a través de muestreos que apunten no ya a conocer solo la diversidad de fuentes en la región, sino también aquellos aspectos tecnológicos y funcionales que permitan entender mejor las estrategias de aprovisionamiento y su variabilidad tanto espacial como temporal en escalas espaciales menores.

\section{AGRADECIMIENTOS}

Deseamos expresar nuestro agradecimiento a Valeria Cortegoso y Ramiro Barberena por la lectura crítica de una versión previa del manuscrito y sus valiosos aportes. A los evaluadores anónimos por sus aportes y comentarios. También agradecemos a los Dres. Víctor Durán, Martín Giesso y Mike Glascock por los análisis efectuados oportunamente y por las respuestas a nuestras frecuentes consultas. Este trabajo se ha realizado en el marco del proyecto PICT IDAC-ICES 610 y PICT 2006/00046.

\section{BIBLIOGRAFÍA}

AMICK, D. S. 1994. Technological organization and the structure of inference in lithic analysis: an examination of Folsom hunting behavior in the American Southwest. In The organization of North American prehistoric chipped stone tools technologies, P. Carr editor, pp. 9-34. Archaeological Series $N^{\circ} 7$ International Monographs in Prehistory, Ann Arbor, Michigan.

ARAGÓN, E. Y N. FRANCO. 1997. Características de rocas para la talla por percusión y propiedades petrográficas. Anales del Instituto de la Patagonia 25: 187-199.

ASCHERO, C. 1975. Ensayo para una clasificación morfológica de artefactos líticos aplicados a estudios tipológicos comparativos. Informe a CONICET, MS.

1893. Ensayo para una clasificación morfológica de artefactos líticos aplicados a estudios tipológicos comparativos. Apéndices A-C. Revisión. Cátedra de Ergología y Tecnología, FFyL-UBA. Buenos Aires, MS.

BAMFORTH, D. B. 1986. Technological efficiency and tool curation. American Antiquity 51 (1): 38-50. 
1991. Technological organization and hunter-gatherer land use. American Antiquity 56: 216-235.

BARBERENA, R., A. HAJDUK; A. F GIL, G. A NEME, V. A DURÁN, M. D GLASCOCK, M. GIESSO, K. BORRAZZO M. P. POMPEI; M L SALGÁN, V. CORTEGOSO, G. VILLAROSA, A. A RUGHINI. 2011. Obsidians in the south-central Andes: geological, geochemical, and archaeological assessment of north Patagonian sources (Argentina). Quaternary International 245:25-36.

BECK, C., A.K. TAYLOR, G. T. JONES, C.M. FADEM, C. R. COOK Y S. A. MILLWARD 2002. Rocks are heavy: transport costs and Paleoarchaic quarry behaviour in the Great Basin. Journal of Anthropological Archaeology 21: 481-507.

BINFORD, L.R. 1979. Organization and formation processes: looking at curated technologies. Journal of Anthropological Research 35 (3): 255-273.

1980 Willow smoke and dog's tails: hunter-gatherer settlement system and archaeological site formation. American Antiquity 45 (1): 4-20.

BORRERO, L. 1994-1995 Arqueología de la Patagonia. Palimpsesto 4:9-69.

2002. Arqueología y biogeografía humana en el sur de Mendoza (Comentario crítico). En: Entre Montañas y Desiertos: Arqueología del sur de Mendoza, A. F. Gil y G. A. Neme editores, pp.: 195-202. Sociedad Argentina de Antropología. Buenos Aires.

CALLAHAN, E. 1979. The Basic of Biface Knapping in the Eastern Fluted Point Tradition. A Manual for Flinknappers and Lithic Analysis. Archaeology of Eastern North American 7 (1):1-180

CHARLIN, J. 2007. Una perspectiva espacial de la intensidad de uso de las materias primas líticas en el campo volcánico Pali Aike (Prov. Santa Cruz, Argentina). En: Arqueología de Fuego-Patagonia. Levantando piedras, desenterrando huesos... y develando arcanos. F., M. Carballo, A. Martinic, Prieto, y G. Bahamonde editores, pp.: 129-150. CEQUA. Punta Arenas, Chile.

2009. Aprovisionamiento, explotación y circulación de obsidianas durante el Holoceno tardío en Pali Aike (Provincia de Santa Cruz). Relaciones de la Sociedad Argentina de Antropología XXXIV: 53-73.

CIVALERO, M. T. Y N. FRANCO. 2003. Early human occupations in Western Santa Cruz Province, Southernmost South America. Quaternary International 130: 77-86.

DE FRANCESCO, A., DURÁN, V., BLOISE, A. Y G. NEME. 2006. Caracterización y procedencia de obsidianas de sitios arqueológicos del área natural protegida Laguna del Diamante (Mendoza Argentina) con metodología no destructiva por fluorescencia de rayos (XRF). Anales de Arqueología y Etnología 61: 53-67.

DURÁN, V. A. 2000. Poblaciones Indígenas de Malargüe. CEIDER/Facultad de Filosofía y Letras, Universidad de Cuyo, Mendoza.

DURÁN V., M. GIESSO, M. GLASCOCK, G. NEME, A. GIL Y L. SANHUEZA. 2004. Estudios de fuentes de aprovisionamiento y redes de distribución de obsidiana durante el Holoceno Tardío en el sur de Mendoza (Argentina). Estudios Atacameños 28: 25-43.

ERICSON, J. E. 1984. Toward the analysis of lithic production system. En: Prehistoric Quarries and Lithic Production, J. E. Ericson y B. Purdy editores, pp.: 1-9.Cambridge University Press. Cambridge.

FLEGENHEIMER N. Y C. BAYÓN 1999. Abastecimiento de rocas en sitios pampeanos tempranos: recolectando colores. En: En los tres reinos: prácticas de recolección en el cono sur de América, C. Aschero, M. Korstanje y P. Vuoto (eds.), pp. 95-107. ED Magna Publicaciones- U. N. de Tucumán, Tucumán

FRANCO, V. N. 2002. ¿Es posible diferenciar los conjuntos líticos atribuidos a la exploración de un espacio de los correspondientes a otras etapas del poblamiento? Revista Werken 3: 119-132. Santiago de Chile, Chile. 2004. La organización tecnológica y el uso de las escalas espaciales amplias. El caso del sur y oeste de Lago Argentino. Temas de Arqueología, Análisis Lítico A. Acosta, D. Loponte y M. Ramos editores, pp.:101-144, Universidad Nacional de Luján, Luján.

2007. Lithic Artifacts and the Information about Human Utilization of Large Areas. En: Artefactos Líticos, Movilidad y Funcionalidad de Sitios: Problemas y Perspectivas, P. Escola y S. Hocsman (eds.), British Archaeological Reports, John and Erica Hedge Ltd., Oxford.

GAMBIER, M. 1985. La Cultura de los Morrillos. Instituto de Investigaciones Arqueológicas y Museo. Facultad de Filosofía, Humanidades y Artes. Universidad Nacional de San Juan, San Juan.

GIESSO, M., DURÁN, V., NEME, G.A., GLASCOCK, M.D., CORTEGOSO, V., GIL, A.F. Y L. SANHUEZA 2011. Application of a Portable XRF to Study Obsidian Use in the Central Andes region of Argentina and Chile. Archaeometry 53 (1): 1-21.

GIL, A. 2000. Arqueología de La Payunia; Sur de Mendoza Tesis Doctoral. Facultad de Ciencias Naturales y Museo. Universidad Nacional de La Plata. MS.

2002. El registro arqueológico y la ocupación humana en La Payunia. En: Entre Montañas y el Desierto: 
Aportes a la Arqueología del Sur Mendocino, A. Gil y G. Neme editores, pp.: 103-118. Sociedad Argentina de Antropología, Serie Libros, Buenos Aires.

2006. Arqueología de la Payunia (Mendoza, Argentina). El poblamiento humano en los márgenes de la agricultura. BAR International Series 1477, Oxford.

GIL, A Y G. NEME 2006. Distribuciones arqueológicas superficiales en Payunia-Llancanelo, En: Arqueología y Ambiente de áreas naturales protegidas de la provincia de Mendoza, Anales de Arqueología y Etnología, V. A. Durán y V. Cortegoso (eds.), Volumen Especial 61: 163-184.

GLASCOCK, M. D., G. E. BRASWELL Y R. H. COBEAN. 1998. A systematic approach to obsidian source characterization. En Archaeological obsidian studies: Method and theory, Advances in archaeological and museum science vol. 3:15-65, M. S. Shackley editor. Plenum Press, Nueva York.

GOULD, R.A. 1978. The anthropology of human residues. American Anthropologist 80: 815-835.

1980 Living Archaeology. Cambridge University Press, Cambridge.

GOULD, R. Y S. SAGGERS 1985. Lithic procurement in Central Australia: a closer look at Binford's idea of embeddedness in archaeology. American Antiquity 50 (1): 117-136.

HISCOCK, P. 2002. Quantifying the size of artifact assemblages. Journal of Archaeological Science 29: 251-258.

INGBAR, E. 1994. Lithic Material Selection and Technological Organization. En: The organization of North American prehistoric chipped stone Technologies, P. Carr editor, pp.: 45-56. International Monographs in Prehistory. Archaeological Series 7, Michigan.

INGBAR, E., M. L. LARSON Y B. BRADLEY 1989. A nontypological approach to debitage analysis. En: Experiments in Lithic Technology, D. Amick y R. Mauldin eds., BAR International Series 528: 117-135. Oxford.

KUHN, S. 1994. A formal approach to the design and assembly of mobile toolkits. American Antiquity 59 (3): 426-442.
2004. Upper Paleolithic raw material economies at Ücagizli cave, Turkey. Journal of Anthropological Archaeology 23: 431-448.

MELTZER, D. 1989. Was Stone Exchange Among Eastern North American Paleoindians? En: Eastern Paleoindian lithic resource use, C. Ellis y J. Lothrop editores, pp.11-39. Westview Press, Boulder.

NAMI, H. 1992. El subsistema tecnológico de la confección de instrumentos líticos y la explotación de los recursos del ambiente: una nueva vía de aproximación. Shincal 2:33-53.

NEME, G. 2002a. Arqueología del Alto Valle del Atuel (Provincia de Mendoza).Tesis Doctoral. Facultad de Ciencias Naturales. Universidad Nacional de La Plata. MS. 2002b. Arqueología del alto valle del río Atuel: modelos, problemas y perspectivas en el estudio arqueológico de las regiones de altura del sur de Mendoza. En Entre Montañas y Desiertos: Arqueología del Sur de Mendoza, A. Gil y G. Neme editores, pp.: 65-84. Sociedad Argentina de Antropología, Serie Libros, Buenos Aires.

2007. Cazadores-recolectores de altura en los Andes meridionales: el alto valle del río Atuel. En: British Archaeological Reports International Series: 1591.

NEME G. Y A. GIL. 2008. Biogeografía humana en los andes meridionales: tendencias arqueológicas en el sur de Mendoza. Chungara, Revista de Antropología Chilena 40 (1):5-18.

ODELL, G. H. 2003. Lithic Analysis (Manuals in Archaeological Method, Theory and Technique). Orser C. E y Shiffer M. B. editores. Springer. New York.

RENFREW, C. 1977. Alternative models for exchange and spatial distribution. En: Exchange Systems in Prehistory, T. Earle y J. Ericson editores, pp. 71-90. Academic Press, New York.

SHACKLEY, M.S. 2005. Obsidian: Geology and Archaeology in the North American Southwest. The University of Arizona Press, Tucson. 
